\title{
Residual Stress Evaluation of Carburized Transmission Steel Gear Using Neutron and Synchrotron X-ray Diffraction and Finite Element Methods
}

\author{
Yoshihisa Sakaida ${ }^{1, a}$, Takanori Serizawa ${ }^{2, b}$, M. Kawauchi ${ }^{3, c}$, and M. Manzanka ${ }^{3}$ \\ ${ }^{1}$ Dept. of Mech. Eng, Shizuoka University, 3-5-1 Johoku, Naka-ku, Hamamatsu, 432-8561 Japan \\ ${ }^{2}$ Grad school of Eng, Shizuoka University, Japan \\ ${ }^{3}$ Yamaha Motor Co., Ltd., Japan
}

atysakai@ipc.shizuoka.ac.jp, ${ }^{\mathrm{b}}$ f0830036@ipc.shizuoka.ac.jp, ${ }^{\mathrm{c}}$ kawauchim@yamaha-motor.co.jp

Keywords: Carburizing, Residual Stress, Neutron, Synchrotron X-ray, Finite Element Method, Transmission Gear, Chromium Molybdenum Steel, Carbon Content, Hardness

\begin{abstract}
A motorcycle transmission gear of chromium-molybdenum steel with $0.2 \% \mathrm{C}$ was carburized in carrier gas. Carburizing process including heating, carburizing, diffusing and quenching was simulated using elastoplastic finite element method. The carbon content, hardness, residual strain and residual stress fields of gear were analyzed. The unstressed lattice plane spacing and residual strains of the interior near the internal spline of gear were experimentally measured by synchrotron x-ray and neutron diffraction methods. As a result, the analyzed carbon content and hardness gradients of gear accorded with the experimental results. The radial, hoop and axial directions of cylindrical gear were found to be not always principal axes of residual stress field. On the other hand, the analyzed residual strains in the radial, hoop and axial directions of gear slightly discorded with the experimental results. Although correlation between the measured three strains was similar to that of the weighted average of analyzed strains, residual strain and stress fields of motorcycle transmission gear could not be accurately predicted at the present finite element analysis. It was concluded that carbon diffusion phenomenon and resultant hardening could be analyzed by the finite element method, and the actual interior residual strain and stress fields should be nondestructively measured by neutron diffraction method.
\end{abstract}

\section{Introduction}

Carburizing process including heating, carburizing, diffusing and quenching is a useful technique for steel parts to harden and strengthen of the surface layer $[1,2]$. The shape of parts, however, changes inhomogeneously after carburizing process because the steel parts such as for motorcycle or motorcar have complicated shape. Almost parts should be machined after carburizing process so that the shape and dimension accuracy of parts is satisfied. Therefore, reduction of the production cost including machining cost is the most important problem that should be settled. In order to control the shape of carburized steel parts, residual stress field built in the parts during carburizing process $[1,3,4,5,6]$ should be understood. Residual stress field near the surface can be measured by x-ray stress measurement $[1,4,7]$. On the other hand, residual stress field that is distributed under hardening layer cannot be measured using $\mathrm{x}$-rays.

Using X-ray stress measurement, biaxial compressive residual stresses are known to be generated on the surface hardening layer of steel parts after carburizing process. The residual stresses of the interior can be expected to become small tension that balanced with surface biaxial compressive residual stresses. In our previous study [5,6], a motorcycle transmission gear was carburized, and then interior residual stress measurement under the surface hardening layer near the internal spline was conducted by neutron diffraction method. Using coupon specimens cut from the same gear, the interior residual strain distributions in the radial, hoop and axial directions were evaluated from 
$\varepsilon=\Delta d / d_{0}$, in which the differences, $\Delta d=d-d_{0}$, in the lattice plane spacing. These interior strains were found to be not always tension and to be distributed complicatedly. By comparing the full widths at half-maximum intensity (FWHM) of neutron diffraction, the interior of gear was found to deform elastically to accommodate the generation of the surface compressive residual stresses because the FWHMs of the interior were relatively wider than that of coupon specimens. However, the residual stress field of the interior could not be evaluated because principal axes of gear were unidentified. For rigorous analysis for the shape control of carburized steel parts, the residual stress state should be caught.

In this study, the carburizing process of the motorcycle transmission gear was first simulated by elastoplastic finite element method. The carbon content, hardness and residual stress states of gear were calculated. Next, carbon content and hardness gradients were measured. The unstressed plane spacing was experimentally determined by synchrotron x-ray diffraction method, and then interior residual strains of gear measured by neutron diffraction method were revaluated. By comparing the experimental results to finite element analysis results, the validity of the residual stress and strain fields of the interior of carburized transmission gear was examined.

\section{Experimental procedure}

The raw material was chromium-molybdenum steel with $0.20 \% \mathrm{C}, 1.02 \% \mathrm{Cr}$ and $0.17 \% \mathrm{Mo}$. In this study, a motorcycle transmission gear was used, as shown in Fig. 1. Transmission gear was carburized at $900{ }^{\circ} \mathrm{C}$ in carrier gas, and then was quenched from $850{ }^{\circ} \mathrm{C}$ in oil bath and tempered at $190{ }^{\circ} \mathrm{C}$. Using cut gear specimen, the carbon content and hardness gradients were measured from the carburized surface to the interior of gear. Carbon content was measured by electron probe x-ray microanalysis (EPMA). Hardness was measured by micro Vickers hardness tester. Indentation load and holding time were $2.9 \mathrm{~N}$ and $15 \mathrm{sec}$., respectively.

Unstressed plane spacing, $d_{0}$, of Fe-110 and 211 planes were experimentally determined using seven reference coupon $[5,6,8]$ specimens, as shown in Fig. 2. In this study, the remaining hardening layer [5] of cubes of $8 \mathrm{~mm}^{3}$ was first removed by emery paper and electro polishing, and then plane spacing, $d_{110}$ and $d_{211}$, was measured by synchrotron x-ray diffraction method. Synchrotron x-ray diffraction was conducted at BL46XU in SPring-8. The wavelength of $\mathrm{x}$-ray was about $0.172 \AA$. Interior residual stress measurement of gear was nondestructively measured by neutron diffraction method. Neutron diffraction was conducted at RESA in JRR-3 of Japan Atomic Energy Research Institute [5,6]. The wavelength of neutron was about $2.07 \AA$ [5]. Diffraction angles near the internal spline were measured by scanning diffracting volume, interior radial, hoop and axial strains were revaluated.

\section{Finite Element Analysis}

Carburizing process of transmission gear was simulated by elastoplastic finite element method using a commercial program DEFORM-HT3 (Yamanaka Engineering Co., Japan). The sixth part of gear was modeled. The model was divided with four-node tetrahedron element as shown in Fig. 3. In this

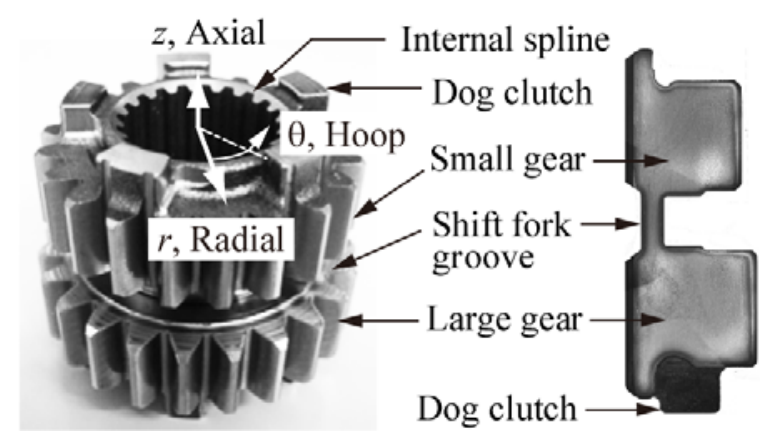

Fig. 1 Transmission gear specimens and definition of cylindrical coordinate system, $r \theta z$.

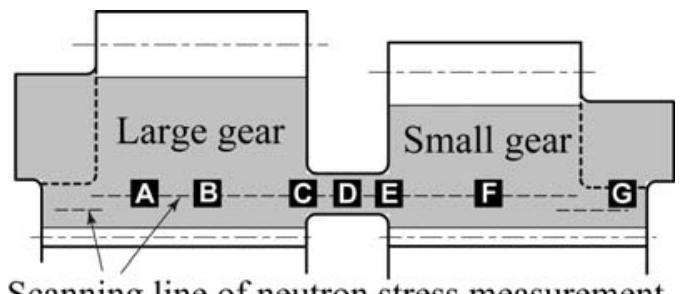

Scanning line of neutron stress measurement

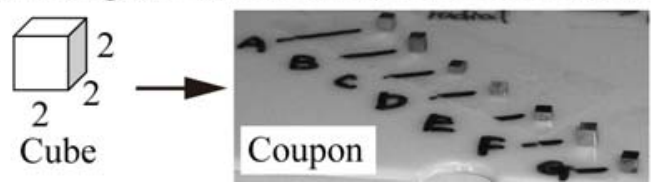

Fig. 2 Reference coupon specimens. 
model, the total number of elements and nodes were 207,662 and 40,460, respectively.

The simulation was carried out step by step with four stages. First stage is heating to $900{ }^{\circ} \mathrm{C}$. Second and third stages are carburizing and diffusing in carrier gas. Forth stage is quenching from $850{ }^{\circ} \mathrm{C}$ in oil bath. In simulation, mechanical properties and thermal properties of chromium-molybdenum steel were attributed to each element as a function of carbon content. The carbon content, hardness, residual stress states were calculated. Interior residual strain distributions in the radial, hoop and axial directions near the internal spline were evaluated by taking the weighted average in the diffracting volume.

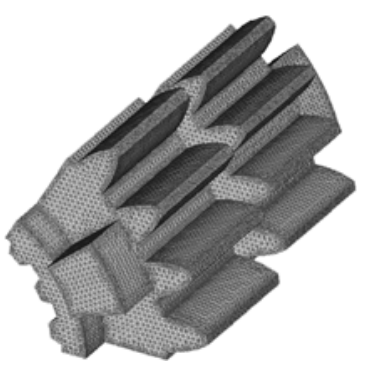

Fig. 3 Finite element model of $1 / 6$ gear.

\section{Results and Discussion}

Figures 4 and 5 show the analyzed carbon content and hardness gradients at large gear and shift fork groove after carburizing process. In these figures, solid and dotted lines indicate the finite element analysis and experimental results. Although the analyzed carbon content at the internal spline or groove outside surfaces and hardness were slightly larger than that of experimental results, the simulation results roughly accorded with experimental results in total. In order to accord the simulation results with the experimental results, hardness database of base materials should be redefined. Carbon diffusion phenomenon and resultant hardening can be simulated by the finite element method.

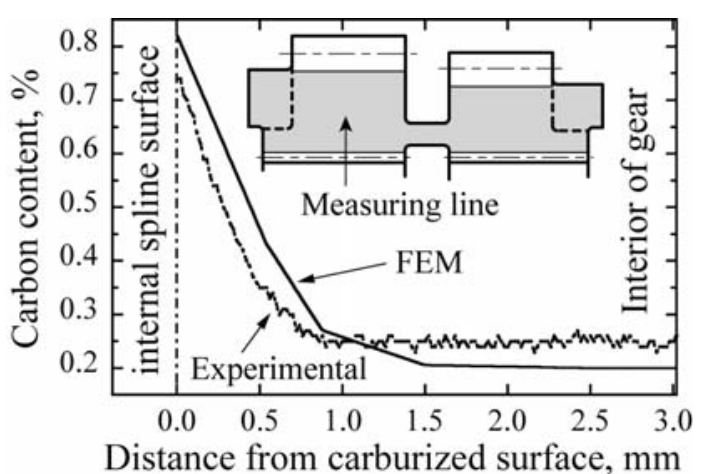

(a) Center of large gear wheel

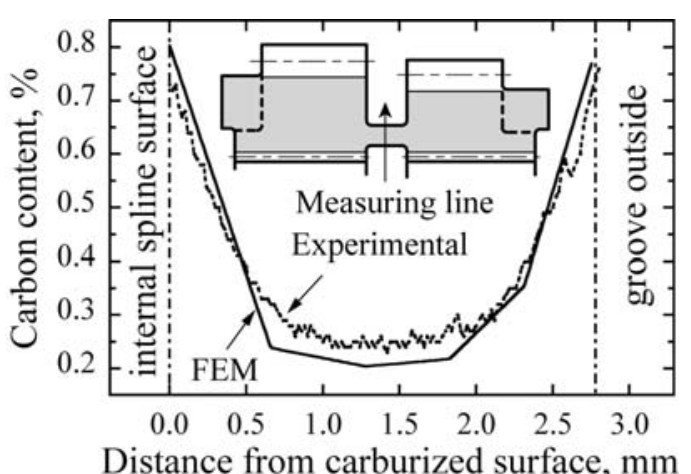

(b) Center of shift fork groove

Fig. 4 Analyzed and measured carbon content gradients at large gear wheel and shift fork groove.

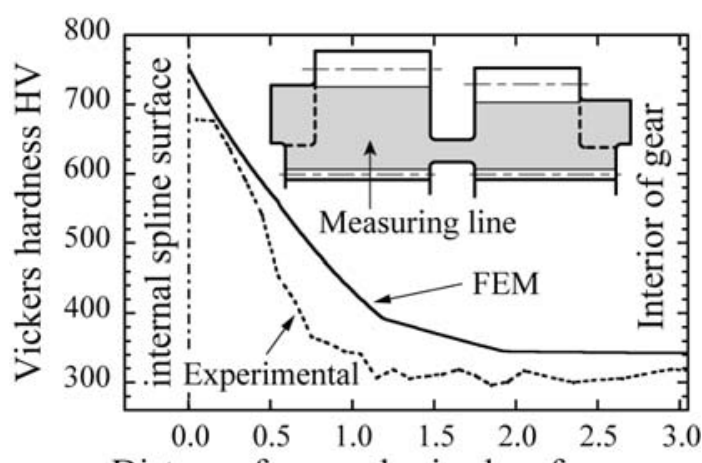

Distance from carburized surface, $\mathrm{mm}$

(a) Center of large gear wheel

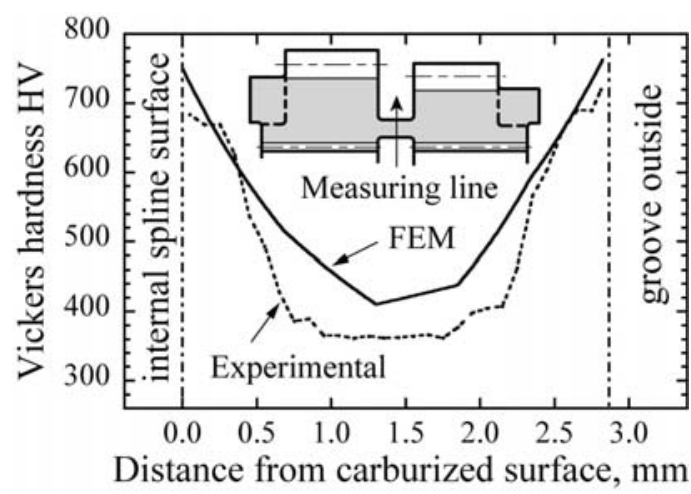

(b) Center of shift fork groove

Fig. 5 Analyzed and measured hardness gradients at large gear wheel and shift fork groove.

Figure 6 shows an example of the analyzed residual stress field in the $\theta$ direction after carburizing process. In spite of the hardening layer of about $0.8 \mathrm{~mm}$ generated over the surface of carburized gear, both the surface and interior residual stresses are distributed complicatedly. Large compressive re- 
sidual stresses are generated near the surface of the both sides of gear wheels and the interior of shift fork groove. Figure 7 shows the analyzed residual stress distributions along the scanning line near the internal spline (as dotted line in Fig.2). In a cylindrical coordinates, three normal stress components, $\sigma_{r}, \sigma_{\theta}$ and $\sigma_{z}$, correspond to the radial, hoop and axial stresses, respectively. Although the absolute values of three shear stress
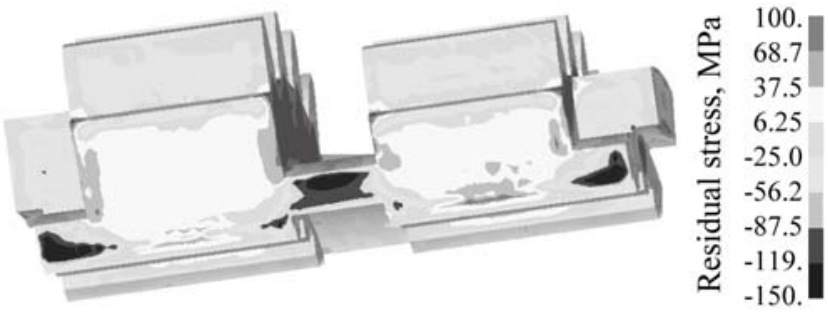

Fig. 6 Hoop residual stress field of transmission gear analyzed by FEM. components, $\sigma_{r \theta}, \sigma_{\theta z}$ and $\sigma_{z r}$, are smaller than that of normal stress components, these values cannot be negligible. Therefore, the radial, hoop and axial directions on the scanning line of neutron stress measurement were found to be not always principal axes of residual stress field of carburized transmission gear. Residual stresses could not be calculated from only these three strains. More than six strains in the different directions have to be measured. Consequently, the radial, hoop and axial strains were picked out of the finite element analysis results, and then were compared with the experimental strains measured by neutron diffraction method.

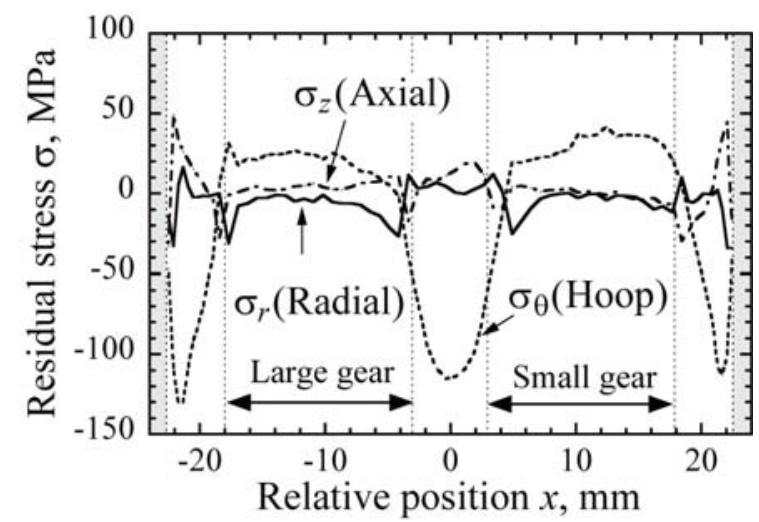

(a) $\sigma_{r}, \sigma_{\theta}$ and $\sigma_{z}$.

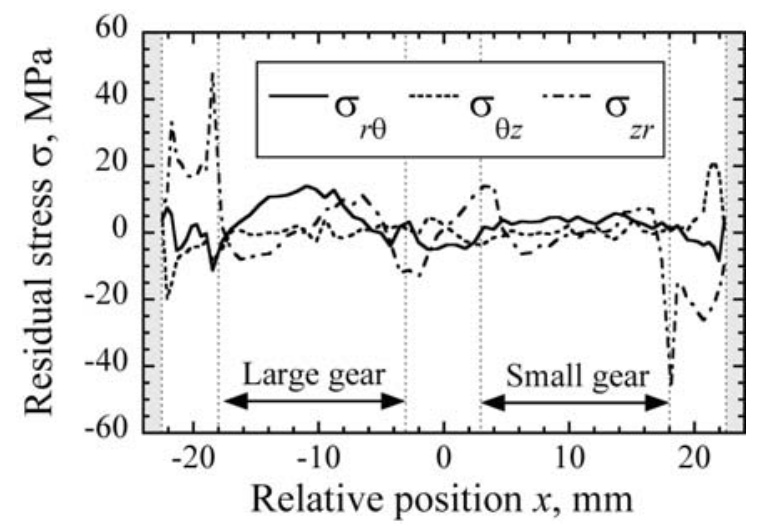

(b) $\sigma_{r \theta}, \sigma_{\theta z}$ and $\sigma_{z r}$.

Fig. 7 Analyzed residual stress fields of motorcycle transmission gear after carburizing process.

Figure 8 shows the picked-out residual strain components, $\varepsilon_{r}, \varepsilon_{\theta}$ and $\varepsilon_{z}$. In the case of hoop strain $\varepsilon_{\theta}$, large compressive residual strains are generated beneath the side surfaces of two gear wheels and within the interior of shift fork groove. On the other hand, tensile residual strains are also generated within the interior of two gear wheels. In this study, the diffracting volume of neutron stress measurement was adjusted by incident and diffracted collimators $[5,6]$. The measured strain was obtained by taking the weighted average of the strains in the diffracting volume. Figure 9 shows the obtained weighted average strains, $\left\langle\varepsilon_{r}\right\rangle_{\text {FEM }},\left\langle\varepsilon_{\theta}\right\rangle_{\text {FEM }}$ and $\left\langle\varepsilon_{z}\right\rangle_{\text {FEM }}$, in the radial, hoop and axial directions. By weighted averaging, steep strain gradients change to the smooth gradients.

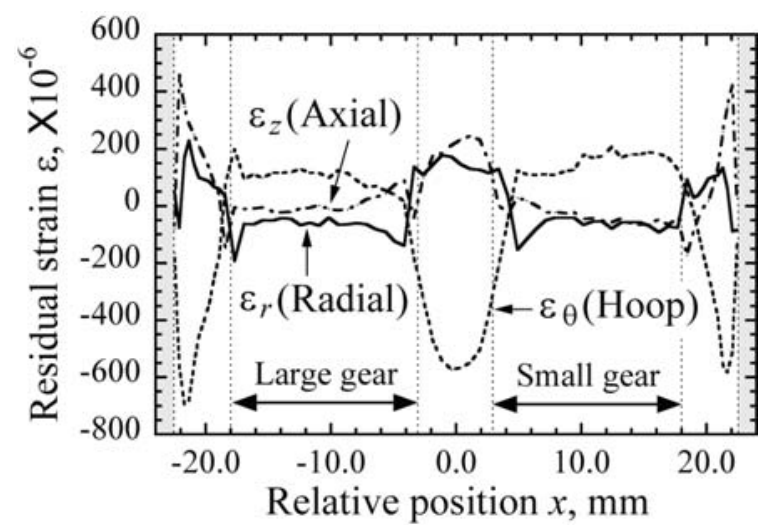

Fig. 8 Analyzed residual strains, $\varepsilon_{r}, \varepsilon_{\theta}$ and $\varepsilon_{z}$.

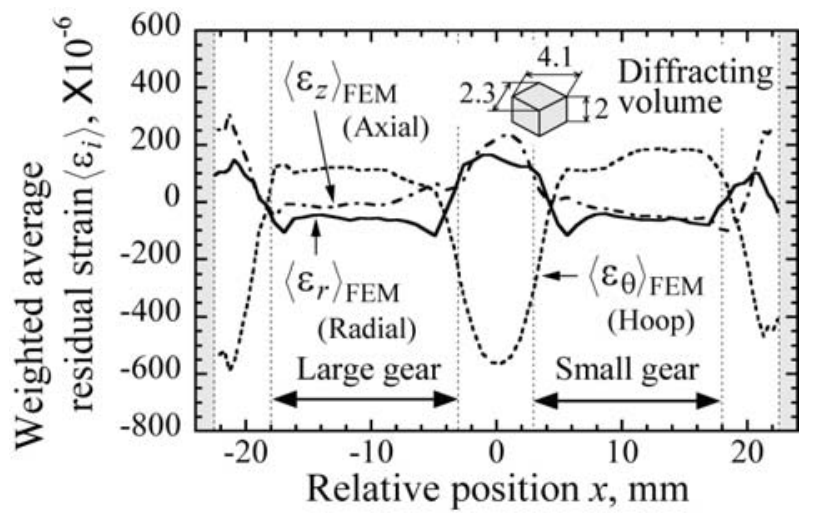

Fig. 9 Weighted average strains, $\left\langle\varepsilon_{i}\right\rangle_{\text {FEM. }}$ 
Figure 10 shows the measured lattice plane spacing of coupon specimens. Each plane spacing of $d_{h k l}$ was determined from the equation, $d_{h k l}=\lambda /\left[2 \sin \theta_{h k l}\right]$. In our previous study [5,6], plane spacing of coupon specimens, C, D, E and G, was relatively changed from the mean value of rest specimens. By removing the remained hardening layer of cut cubes, the plane spacing of coupon specimens was almost equaled, and then was redefined as the measured unstressed ones, $d_{0, h k l}$.

Figure 11 shows the revaluated residual strain distributions, $\left\langle\varepsilon_{r}\right\rangle_{\text {Exp. }},\left\langle\varepsilon_{\theta}\right\rangle_{\text {Exp. }}$ and $\left\langle\varepsilon_{z}\right\rangle_{\text {Exp., along }}$ the scanning line of carburized gear. By comparison between the revaluated and the weighted average residual strains, the correlation between the measured three strains, $\left\langle\varepsilon_{i}\right\rangle$ Exp., was similar to that of the weighted average strains, $\left\langle\varepsilon_{i}\right\rangle_{\text {FEM }}$. It was thought that the difference between $\left\langle\varepsilon_{i}\right\rangle_{\text {Exp. }}$ and $\left\langle\varepsilon_{i}\right\rangle_{\text {FEM }}$ of each strain arose from the difference between the actual and simulated boundary conditions.

Next step, in order to accord the experimental results, $\left\langle\varepsilon_{\theta}\right\rangle_{\text {Exp. }}$ and $\left\langle\varepsilon_{z}\right\rangle_{\text {Exp., with }}$ the simulation results, $\left\langle\varepsilon_{\theta}\right\rangle$ FEM and $\left\langle\varepsilon_{z}\right\rangle$ FEM, the optimum unstressed plane spacing, $d_{0}$, Opti., was determined by a least squares method. Table 1 summarizes the optimized unstressed plane spacing of Fe-110 and 211 planes. In this table, the measured unstressed plane spacing was showed as $d_{0 \text {, Exp., }}$ (as solid and dotted lines in Fig.10). The optimum unstressed plane spacing of $\mathrm{Fe}-211$ plane was very close to the experimental value. On the other hand, the optimum unstressed plane spacing of $\mathrm{Fe}-110$ plane slightly deviated from the experimental value.

Figures 12 and 13 show the residual strains,

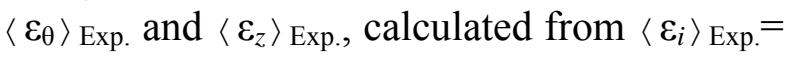
$\Delta d / d_{0}$, Opti. The interior residual strains revaluated from the neutron diffraction angles were found to accord with the weighted average strains by optimizing unstressed plane spacing. Conversely, residual strain and stress fields of transmission gear can't be accurately predicted at the

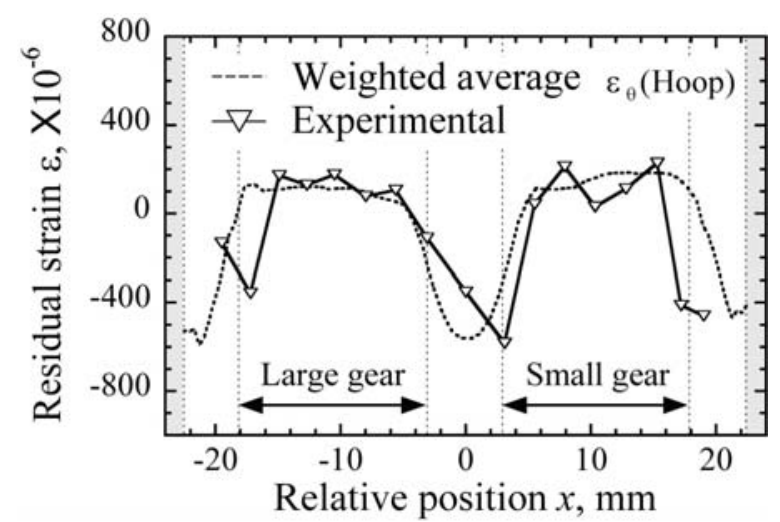

Fig. 12 Comparison between simulation and experimental results for $\varepsilon_{\theta}$ (Hoop).

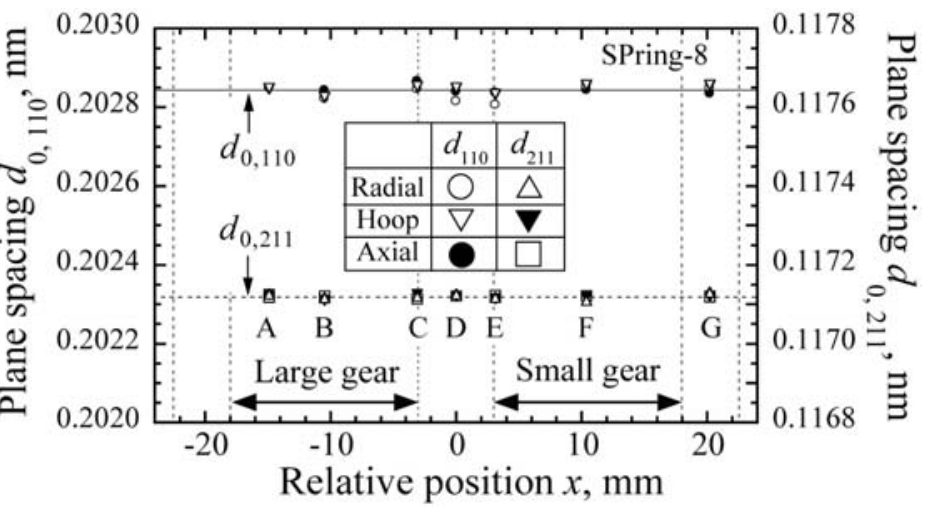

Fig. 10 Lattice plane-spacing of coupon specimens.

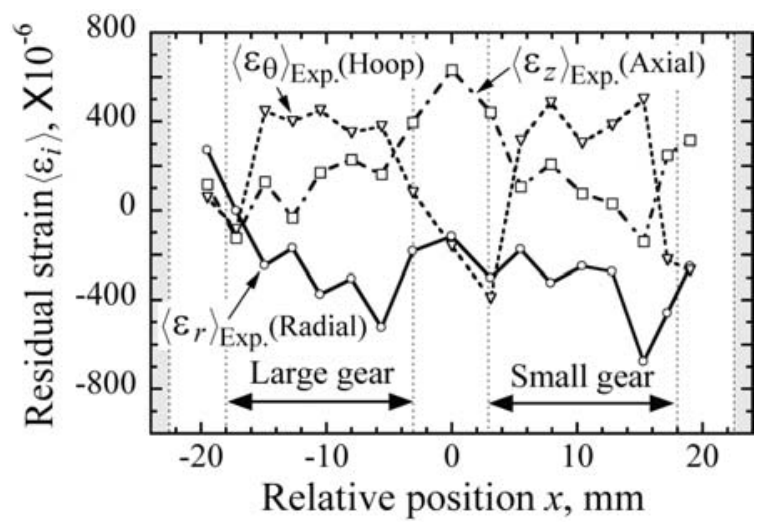

Fig. 11 Measured residual strains, $\varepsilon_{r}, \varepsilon_{\theta}$ and $\varepsilon_{z}$.

Table 1. Optimized plane spacing, $d_{0 \text {, Opti. }}$ for Fe-110 and 211 Planes.

\begin{tabular}{l|c|c}
\hline Diffraction & 110 & 211 \\
\hline$d_{0, \text { Exp. }}(\mathrm{nm})$ & 0.20284 & 0.11712 \\
\hline$d_{0, \text { Opti. }}(\mathrm{nm})$ & 0.20290 & 0.11714 \\
\hline
\end{tabular}

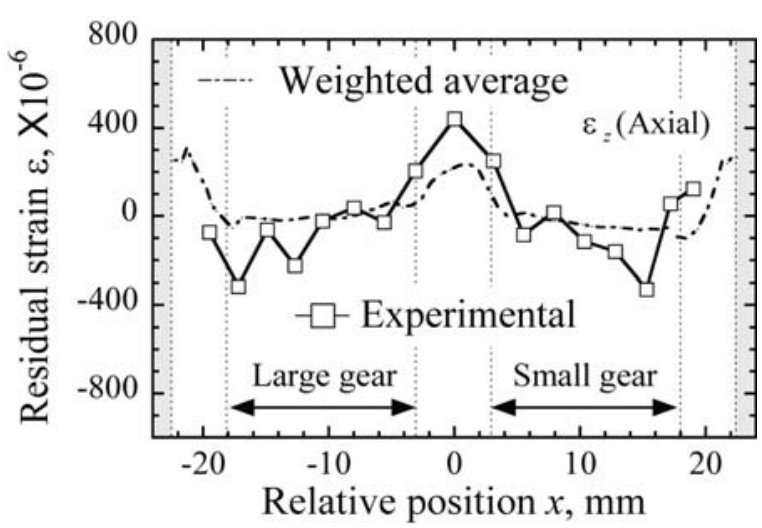

Fig. 13 Comparison between simulation and experimental results for $\varepsilon_{\mathrm{z}}$ (Axial). 
present finite element analysis. The actual interior residual strain and stress fields should be nondestructively measured by neutron diffraction method. It is also important to establish an accurate method of measuring or evaluating unstressed lattice spacing, because the estimate of unstressed plane spacing directly affects the result of residual stress measurement.

\section{Conclusions}

The carbon content, hardness and residual stress fields of a carburized motorcycle transmission gear was analyzed by elastoplastic finite element method. The unstressed plane spacing and residual strains of the interior of gear were experimentally evaluated by synchrotron x-ray and neutron diffraction methods. The results are summarized as follows:

(1) The analyzed carbon content at the internal spline or groove outside surfaces and hardness were slightly larger than that of experimental results. The simulation results roughly accorded with experimental results in total. Carbon diffusion phenomenon and resultant hardening could be analyzed by the finite element method.

(2) From the analyzed residual stress field, both the surface and interior residual stresses were distributed complicatedly in spite of the hardening layer of about $0.8 \mathrm{~mm}$ generated over the surface. Large compressive residual stresses were generated near the surface of gear wheels and the interior of shift fork groove. The radial, hoop and axial directions of transmission gear were found to be not always principal axes.

(3) The analyzed residual strains picked out in the radial, hoop and axial directions slightly discorded with the experimental results. Correlation between the measured three strains was similar to that of the weighted average of analyzed strains.

(4) Residual strain and stress fields could not be accurately predicted at the present finite element analysis. The actual interior residual strain and stress fields should be nondestructively measured by neutron diffraction method.

\section{Acknowledgment}

Synchrotron x-ray and Neutron diffractions were performed with the approval of the Japan synchrotron radiation research institute (No. 2008B2030) and Neutron trial use program (No. 26 and 48). This work was partially supported by Grant-in-Aid for Scientific Research (C), No. 21560087. The authors thank Dr. T. Shobu, Dr. H. Suzuki (JAEA), Dr. M. Hayashi (Ibaraki Prefectural Govt.) and Y. Mizukami (graduate student of Shizuoka Univ.) for experimental support and Dr. K. Hayakawa (Shizuoka Univ.) for helpful advice to finite element analysis.

\section{References}

[1] P. Rangaswamy, C.P. Scherer, M. A. M. Bourke, Mater. Sci. and Eng, A298 (2001), 158.

[2] O. Asi, A. C. Can, J. Pineault, and M. Belassel, Surface \& Coatings Technology, 201 (2007), 5979.

[3] Q. Yang, X. Ren, Y. Gao, Y. Li, Y. Zhao and M. Yao, Mater. Sci. and Eng, A 392 (2005), 240.

[4] M. A. M. Bourke, P. Rangaswamy, T. M. Holden and R. Leachman, Mater. Sci. and Eng, A257 (1998), 333.

[5] Y. Sakaida, M. Kawauchi and M. Manzanka, Advances in X-ray Analysis, 52 (2009), 561.

[6] Y. Sakaida, M. Kawauchi, M. Manzanka, H. Akiyoshi, H. Suzuki and T. Saito, Trans. Jpn. Soc. Mech. Eng., A 74-740 (2008), 598.

[7] I. C. Noyan and J. B. Cohen, Residual Stress; Measurement by Diffraction and Interpretation, Springer-Verlag: New York, (1987), p. 69.

[8] T. M. Holden, H. Suzuki and D. G. Carr, ISIJ International, 46-7 (2006), 959. 